\title{
PLAN ESTATAL DE VOLUNTARIADO 2001/2004
}

\section{TÍTULO I: DECLARACIÓN PRELIMINAR}

El voluntariado es una pieza fundamental para la construcción de la sociedad civil. Da vida a las aspiraciones más nobles de la humanidad: la búsqueda de la paz, la libertad, la oportunidad, la seguridad y la justicia para todas las personas.

- En esta época de globalización y de cambio continuo, el mundo se está haciendo cada vez más pequeño, más interdependiente y más complejo. El voluntariado, tanto a través de la acción individual como de la grupal, es una forma de:

- Poder sostener y fortalecer los valores humanos de comunidad, atención y servicio.

- Permitir a los individuos que ejerzan sus derechos y responsabilidades como miembros de sus comunidades, aprendiendo y creciendo a lo largo de toda la vida, desarrollando todo su potencial humano.

- Así como establecer contactos, dejando a un lado las diferencias que nos separan, de modo que podamos convivir en comunidades saludables y sostenibles, trabajando juntos para proporcionar soluciones innovadoras a nuestros desafíos comunes y para compartir nuestro destino colectivo.

- En el despertar del nuevo milenio, el voluntariado es un elemento esencial de todas las sociedades. Convierte en acción práctica y efectiva la declaración de Naciones Unidas de que «Nosotros, Los Pueblos» tenemos el poder de cambiar el mundo.

(Sección 1 de la Declaración Universal del Voluntariado adoptada por 14VE, Países Bajos, enero 2001)

\section{TÍTULO II: PRESENTACIÓN DEL PLAN ESTATAL 2001/2004}

- La conclusión del Plan Estatal del Voluntariado 1997/2000, así como la aceptación que ha tenido su desarrollo, principalmente entre Comunidades Autónomas y ONG de acción social, han dado lugar a la oportunidad de elaborar un nuevo Plan, en cuya redacción se han considerado varios aspectos.

- Por una parte, se ha de tener en cuenta el hecho de que es difícil incidir en un movimiento social, como es el movimiento voluntario, y conocer cuál es su situación en cada momento, debido a su complejidad, en cuanto que afecta a sectores muy diversos de la vida en comunidad, y debido a que, en general, toda evolución social requiere una plazo de tiempo bastante amplio para mostrar claramente sus frutos. Ello supone la necesidad de actuar estratégicamente, es decir, priorizando ciertos sectores de la realidad y con objetivos a corto plazo. Para trazar las líneas que alcancen estos objetivos, se ha de partir de cuál es el entorno en que se ha de implementar este Plan, y cuáles han sido sus logros y debilidades, con el fin de recoger aquellas actuaciones que permitan a los sectores implicados adaptarse a las oportunidades y desafíos de dicho entorno, aquéllas que profundicen en los logros del Plan anterior y las que aporten nuevas sugerencias en los ámbitos en que los resultados han sido más pobres.

- Además, se ha de llevar cabo una valoración periódica de cómo se está ejecutando el Plan y una final sobre el grado de su desarrollo y sobre la incidencia que pueda tener 
a su conclusión, determinando la consecución o no de los objetivos operativos propuestos y dando a conocer qué proyectos y experiencias se han realizado en virtud de las sugerencias y actuaciones contenidas en el Plan. Por ello, se incluye un último capítulo en que se esboza la metodología y los plazos en que se han de llevar a cabo el seguimiento y la evaluación del Plan 2001/2004.

\section{TÍTULO III: MARCO SOCIAL Y ADMINISTRATIVO DEL PLAN}

Marco Social

- A la luz de este planteamiento, cabe hacer mención, en primer lugar, de cuáles son las principales tendencias que han de influir necesariamente sobre la configuración del entramado social en estos próximos cuatro años y su posterior evolución, tendencias en que se ven implicados diversos factores de tipo político, sociocultural o económico. Estos factores de cambio tendrán, sin duda, una importante incidencia sobre aspectos diversos de las ONG (las demandas sociales, los servicios con que respondan a ellas, sus formas de organización o captación de fondos), lo que reviste una importancia esencial.

- Entre los factores políticos cabe señalar tres aspectos: la crisis de las ideologías, la fragmentación del poder del Estado, redefinición del papel que las Administraciones Públicas han de desempeñar y, por ende, el de los demás actores sociales.

- Uno de los fenómenos políticos más significativos en la actualidad es lo que se ha dado en llamar el fin de las ideologías, la difuminación de fronteras entre ideas, métodos o políticas que tradicionalmente se identificaban como de derechas o de izquierdas, y, en consecuencia, la intensificación de las relaciones entre grupos y organizaciones de diferente signo.

- Ello puede hacer pensar en una desvinculación de la labor de las ONG con el mundo religioso (de acuerdo con el documento «Las organizaciones de Voluntariado en España» de la Plataforma para la Promoción del Voluntariado en España, 1997, aún entonces en nuestro país el 23 '7\% de las entidades eran de carácter confesional), así como en un claro acercamiento de perspectivas desde las que actuar y de necesidad sociales atendidas por las ONG, y en consecuencia, una mayor cooperación entre ONG para solucionar de forma integral los problemas sociales, proceso que no está consolidado en nuestro país (según datos del mismo documento sólo el 52 '5\% de las ONG se encuentra integrado en otra, en una federación o en una coordinadora).

- Otro factor significativo es la fragmentación del poder del Estado, de un lado, dado por la internacionalización de la política, fenómeno que tiene una importancia mayor, si cabe, en el caso español, ya que España pertenece a la Unión Europea, una organización supranacional que ejerce una parcela de soberanía cedida por los Estados miembros, y, de otro lado, dado por el proceso de descentralización política que se vive en muchos países, origen en el nuestro de las Comunidades Autónomas. Paralelamente, han nacido nuevas instancias revestidas asimismo de poder, de tipo económico o comunicacional, lo que viene significando una paulatina pérdida del poder estatal, de su autonomía, de su prestigio y de su capacidad de actuación.

- Esta fragmentación puede verse, paradójicamente, como una multiplicación del poder y con él, una importante ampliación de la labor administrativa, de su producción jurídica, de las fuentes de financiación para las entidades, y de todas aquellas actuaciones o servicios prestados por las instancias públicas que pueden beneficiar, en gran medida, la labor de la iniciativa social. Ello supone una importante intensi- 
ficación de las relaciones entre ONG y Administraciones Públicas y, no sólo Administraciones nacionales, sino también diferentes organismos administrativos de carácter internacional.

- La terecra tendencia citada es la redefinición del papel del Estado, ya que del Estado de Bienestar, responsable exclusivo de la provisión de servicios sociales a los ciudadanos, se ha pasado a un nuevo esquema de relaciones entre el Estado y la Sociedad Civil, Io que los expertos han dado en llamar el Estado Relacional o Sociedad del Bienestar, que se ha traducido en una mayor corresponsabilidad en la satisfacción de las necesidades sociales de todos los sectores, creando nuevas sinergias y recreando las formas tradicionales de participación ciudadana.

- Ello supone que tanto las entidades empresariales como las organizaciones no lucrativas son también responsables de esa cobertura social, por sí o conjuntamente, lo que principalmente se encauza por diversos medios, entre los que destaca el marketing social, ya que se estima que en España está moviendo unos 20.000 millones anuales, aunque, como las otras formas de relación, tropieza con la polémica planteada en torno a los verdaderos motivos de una empresa (si son éticos o únicamente comerciales) y el hecho de que en esa polémica se vean también involucradas $O N G$, polémica cuya resolución pasa a ser de especial importancia para lograr una máxima colaboración entre entidades y un importante apoyo a las ONG por las empresas.

- Desde la perspectiva sociocultural, cabe destacar los cambios demográficos, culturales y educativos, también esenciales en la configuración de la sociedad.

- La población es uno de los factores clave en la definición de los distintos ámbitos de atención pública, dado que gran parte de las necesidades y las demandas de una sociedad vienen determinadas por ella. En España y en el conjunto internacional se vienen produciendo importantes cambios demográficos: la progresiva disminución de nacimientos (según datos del Atlas del Banco Mundial / Informe sobre Desarro1lo Mundial 2000-2001 la tasa de crecimiento anual en España cs de 0'2\%), una mayor esperanza de vida (78 años, de acuerdo con esa misma fuente), el envcjecimiento de la población (el Anuario Social de España 2000, de la Fundación La Caixa, recoge que el 16’3\% de la población española tiene más de 65 años) o la entrada de inmigrantes desde países menos desarrollados, hoy habitualmente varones en edad de trabajar.

- La problemática que estos cambios conllevan, de atención social a las personas mayores, cobertura de su tiempo de ocio o de ayuda a la integración cultural de los inmigrantes, se va a agravar en los próximos años, e incluso puede dar lugar a consccuencias inesperadas, y las ONG están también llamadas a responder a esa nueva problemática por su propio carácter, porque son más flexibles y creativas en sus modos de actuar, y porque atienden cada situación desde una perspectiva global e integradora.

- En segundo lugar, es un factor muy favorable para la vigorización de la cultura de solidaridad la difusión de una serie de nuevos valores promovidos por diferentes hechos sociales, como la urbanización (según el citado Atlas el 76\% de la población española es urbana) y la imposición de la comunicación social y las nuevas tecnologías.

- Aquellos valores van desde el mayor respeto hacia el medio ambiente (según el documento de la citada Plataforma, el 2'8\% de las ONG trabaja en este campo), 
hasta la vuelta a los principios liberales clásicos de fraternidad, igualdad, la justicia social, o la participación de los ciudadanos en todos los temas de interés general, la concienciación sobre las diferencias Norte-Sur, o la apreciación del ocio.

- Estos valores no sólo ayudan a legitimar la labor de las ONG, sino que también abren nuevos campos de demanda social y de acción para cllas, bien por una carencia objetiva, como la cooperación al desarrollo, bien por el deseo de mejorar la calidad de vida de los ciudadanos, como el uso del tiempo libre. Para cllo, es cspecialmente significativa la realización de campañas de sensibilización, principalmente a través de los medios de comunicación social, sobre necesidades. que apenas quedan cubiertas, como la atención a ciertas áreas o colectivos marginados.

- Por último, no se ha de olvidar la importancia de la elevación de los niveles educativos y de especialización profesional, que se viene dando en todas las sociedades que está producida tanto por consideraciones de tipo político y humanitario, como por exigencias del sistema productivo, que cada vez más requiere trabajadores con una mayor formación general y específica.

- Ésta es quizá una de las grandes desventajas de las ONU en comparación con el Sector Público o Privado, porque, en términos generales, no pueden ofrecer grandes compensaciones retributivas o laborales a sus colaboradores. Por ello, se han de esforzar por mantener el atractivo de un trabajo desinteresado para continuar captando a personas cualificadas tanto en áreas sociales tradicionales, como en nuevas áreas de gestión y dirección de organizaciones, de modo que no se abra una brecha infranqueable entre la calidad de los servicios que ellas prestan y la de los servicios ofrecidos por los otros dos sectores. Además, no se ha de olvidar que los recursos humanos de las entidades no lucrativas y, en particular, el voluntariado, constituyen, precisamente, la esencia misma de las ONG y son los que dan sentido a su forma de trabajar y a los proyectos que llevan a cabo.

- En cuanto a las tendencias económicas, destacan cuatro: la internacionalización de la economía, la agudización de la competencia, el creciente protagonismo dentro del sistema productivo de los servicios y diversas transformaciones en el mercado laboral.

- Uno de los cambios, no sólo económico, sino también político y cultural que se viene dando en los últimos años y que se acrecentará en los venideros, es la internacionalización de la economía, la intensificación de las relaciones comerciales, financieras o monetarias en el conjunto mundial, influida por los avances tecnológicos, los transportes y las comunicaciones, como muestra el hecho de que el comercio mundial mueve en torno a 5.500.000.000 de millones de dólares, de acuerdo con cl Informe Anual 2000 de la Organización Mundial de Comercio.

- En este entorno complejo y global, la coordinación entre ONG, a nivel nacional e internacional, no sólo ayudará a una mejora de la gestión de cada una, sino que además puede ser la única vía de solucionar problemas mundiales, como la cooperación con los países menos desarrollados (35 países tienen un desarrollo humano bajo, según el Informe sobre Desarrollo Humano 2000 del PNUD), la conservación del medio ambiente, con toda la problemática que supone el cambio climático (desertización, deforestación, emisión de gases nocivos a la atmósfera) o los flujos humanos de inmigrantes o refugiados (hay en la actualidad más de 22 millones de personas bajo el amparo del ACNUR). De este modo, la internacionalización pasa a ser tanto una oportunidad de intercambio y aprendizaje para las $O N G$, como un ingente campo de trabajo. 
- En segundo lugar, se ha de mencionar la imposición de la competencia entre las entidades por los recursos y por la demanda, tanto a nivel internacional como nacional, que hace que las organizaciones se esfuercen por ser más eficaces y eficientes en todos sus procesos.

- Si bien el principio de la competitividad, llevado hasta sus últimas consecuencias, no debe ser aplicado al Tercer Sector, sí lo son muchos de los principios y medios que emplean las empresas para ser competitivas. Las ONG deben también ser eficaces y eficientes y, para ello, se han de esforzar por diversificar sus vías de financiación, por dar a conocer a la comunidad su contribución al bienestar y sus logros, han de crear estructuras de coordinación para hacer presión sobre los gobiernos, para plantear sus problemas y conocer las experiencias de otras ONG en la resolución de problemas semejantes.

- En cuanto a la terciarización, sin duda es el sector servicios el que tiene una mayor aportación al Producto Interior Bruto y a la creación de riqueza en las sociedades occidentales, Producto que, según las Perspectiva Económicas de la OCDE, 2000, creccrá un 2'6\% en los años 2002-2006.

- Ésta cs una gran oportunidad para el Tercer Sector (de acuerdo con los datos del estudio de la Fundación BBV, aporta en torno al 6 ' $5 \%$ del Producto español) y, en particular, para las ONG, ya que en la actualidad se consideran como uno de los sectores con más potencial futuro en la creación de riqueza, y precisamente en el sector servicios, y como uno de los principales yacimientos de empleo.

- En cuanto al mercado de trabajo, se están produciendo importantes cambios cuantitativos, referidos a la reducción de la vida laboral de las personas y la disposición de un mayor tiempo libre, y cualitativos, por la necesidad de perfiles profesionales diferentes y la creación de puestos en nuevos sectores o ámbitos.

- En la actualidad, los colectivos que en mayor medida colaboran como voluntarios son, precisamente, aquéllos que quedan fuera del mercado de trabajo, desempleados, estudiantes o jubilados. Paralelamente, el gran reto es el de saber dar a conocer a los trabajadores la posibilidad de emplear su tiempo libre de forma útil y solidaria. Por otra parte, de acuerdo con el estudio «Empleo y trabajo voluntario en las ONG de acción social», de la Fundación Tomillo, 2000, las ONG constituyen un elemento esencial de creación de empleo, principalmente las organizaciones más recientes y las de mayor tamaño. Se calcula que las ONG contratarán, en los años 2000-2001, a casi 50.000 asalariados, principalmente en las áreas de servicios de restauración, personales, protección social y comercio. Esto permitirá a dichas entidades contar con una dotación humana adecuada a las funciones y proyectos que tengan en marcha.

- En resumen, se pueden señalar las siguientes conclusiones consideradas en la elaboración del Plan:

- Una vía esencial para lograr un máximo acercamiento y entendimiento entre las ONG de diferente signo consiste en la intensificación de las relaciones en el marco del propio Tercer Sector.

- Las Administraciones Públicas han de ofrecer todo su apoyo a las ONG y al voluntariado para que desempeñen adecuadamente el papel social que les correspondc.

- Empresas privadas y entidades sin ánimo de lucro han de corresponsabilizarse, junto a los poderes públicos, de la resolución de los problemas sociales. 
- Las ONG han de dar cabida a todos aquellos ciudadanos que acudan a ellas bien en busca de atención para sus necesidades específicas, como beneficiarios, bien con el fin de participar en el trabajo social que las entidades realizan, como voluntarios.

- En ese sentido, se ha de dar la información precisa a la sociedad, de la forma más amplia y completa posible, para que los ciudadanos puedan optar por esta forma de participación, en pos de la consecución de valores como los de solidaridad, justicia o fraternidad.

- Se ha de seguir avanzando en los diversos aspectos vinculados con la educación en valores de la infancia y la juventud, y en la formación de los trabajadores de las ONG, sean profesionales, sean voluntarios.

- La información e integración en redes y organismos internacionales puede ser la única vía de solucionar muchos de los problemas mundiales.

- La aplicación de sistemas o principios propios de la empresa constituye una vía muy válida para la mejora y la modernización de las ONG.

- Se ha de apoyar a las organizaciones de voluntariado para que refuercen sus estructuras y cuenten con los recursos precisos para desarrollar al máximo sus potencialidades en la prestación de los servicios que les son propios, dando respuestas innovadoras a los problemas sociales y afianzando el compromiso ciudadano, en la creación de riqueza y para explotar todas sus posibilidades como yacimientos de empleo.

\section{Marco Administrativo}

- La elaboración del Plan Estatal del Voluntariado 2001/2004 exige, junto a la consideración de las actuales tendencias sociales, políticas y económicas, y de cómo han de incidir éstas en la configuración del Tercer Sector, la inclusión de datos y consideraciones provenientes del Balance del anterior Plan así como de las aportaciones e ideas de cuantas instancias se hayan implicadas en esta materia.

- En primer lugar, se ha tratado de reunir en la redacción del Balance del Plan 1997/ 2000 dos facetas distintas de igual importancia: la referida a la ejecución de las medidas del Plan y la relativa a su impacto.

- Por una parte, de las 127 actuaciones del Plan, se han desarrollado 92 (72'44\%) con 797 proyectos. En particular, cabe destacar la importancia de lo realizado en materia de sensibilización, pues el 92'3\% de las actuaciones propuestas inicialmente tuvicron desarrollo, y, por el contrario, la escasa significación del número de proyectos acometidos en materia de coordinación, dado que sólo el $50 \%$ de las actuaciones se han ejecutado. En cuanto al aspecto financiero, no es fácil dar unas cifras definitivas, pero considerando sólo la que ha sido aportada por las entidades promotoras de los proyectos, el presupuesto destinado en esos años al voluntariado asciende, al menos, a 5.100 millones de pesetas (5.100.061.239).

- Por otra parte, la valoración cualitativa sobre el verdadero impacto del Plan, junto a otros factores, sobre el movimiento voluntario los últimos años, se recoge básicamente en el apartado del Balance dedicado a los puntos fuertes y las debilidades de la ejecución del Plan 1997/2000.

- Los logros o puntos fuertes que se señalan en el Balance son los siguientes:

- Su propia existencia y singularidad, la redacción de sus objetivos y áreas, y la puesta en marcha de sus actuaciones o proyectos.

- La metodología seguida en su elaboración y ejecución, ya que siempre se ha 
buscado el consenso y el protagonismo de las ONG y los voluntarios que colaboran en cllas, dando al sector un documento de sugerencias y propuestas que le facilite la realización de una labor autónoma y actuando bajo el signo de la participación, que reúna perspectivas diferentes pero complementarias.

- Ha sido útil para vigorizar el voluntariado como forma de participación y compromiso social de los ciudadanos, ayudando a aplicar plenamente los contenidos de la Ley 6/1996 del Voluntariado.

- En materia de sensibilización, área que, como queda dicho, es la que se ha desarrollado en mayor medida, destacan varios logros:

- El crecimiento del número de voluntarios, de programas de voluntariado y de ONG que se ha producido en los últimos años, aunque, por supuesto, se debe también a otros muchos factores.

- Cambios en el perfil del voluntario, ya que cada vez son más los mayores y las personas ajenas al entorno familiar del beneficiario, quienes se interesan en las labores de acción social, se trabaja más en ámbitos territoriales sin una tradición asociativa significativa, y la responsabilidad voluntaria y su compromiso son cada vez más fuertes.

- En cuanto a la promoción, destacan una serie de hechos:

- El Plan ha ayudado a las entidades menores o más jóvenes a gestionar programas de voluntariado (de información a la sociedad, de coordinación entre entidades que trabajan en los mismos ámbitos, consolidación de recursos...) y a la vigorización de valores y principios eminentemente participativos en sus estructuras y en su actuación.

- En relación con ese tema, se ha conseguido una mayor consideración de los propios voluntarios en la jerarquía de la ONG, creando departamentos de gestión global de recursos humanos, y definiendo los roles de cada tipo de personal (voluntarios y profesionales, objetores o cooperantes).

- Se ha dado importancia al reconocimiento individual de los voluntarios, con la promoción del voluntario en el marco de cada una de las organizaciones.

- En muchas instituciones y servicios públicos, se han abierto nuevos espacios para la participación ciudadana y de las $O N G$ en la gestión y dirección.

- Ha supuesto el aumento del interés político y, por consiguiente, de la acción administrativa hacia el fomento del voluntariado y de la participación social.

- En materia de apoyo y dotación a las entidades no lucrativas, el Plan Estatal 1997/ 2000 ha significado diversas mejoras:

- Incremento del crédito destinado a programas de voluntariado en las últimas convocatorias con cargo a la asignación tributaria del Impuesto sobre la Renta de las Personas Físicas gestionada por el Ministerio de Trabajo y Asuntos Sociales, de los 491 millones de pesetas distribuidas en 1996 o los casi 588 del año 1997, hasta los 777 millones de pesetas ( 777.825 .029 pesetas) para el año 2000; incremento que, necesariamente, ha supuesto una mejora significativa en la prestación de servicios y en la realización de programas para el voluntariado en las $O N G$.

- Importancia concedida a los proyectos de formación de los voluntarios, gestores de voluntariado y miembros de las $O N G$ en general.

- Finalmente, los logros del Plan anterior en materia de coordinación han sido los siguientes:

- Diálogo inicial entre ONG y Administración centrado en aspectos económicoadministrativos y legislativos, uno de cuyos principales resultados ha sido la 
creación del Consejo Estatal de ONG de Acción Social.

- Creación de estructuras y movilización de recursos en el marco de las Administraciones Públicas, y en particular, creación de un crédito en el presupuesto del Ministerio de Trabajo y Asuntos Sociales que se transfiere a las Comunidades Autónomas y las Ciudades de Ceuta y Melilla para la ejecución de proyectos en desarrollo del Plan, crédito que en el año 2000 alcanzó los 400 millones, y al que se ha de sumar lo aportado por las Administraciones Territoriales, dado que existe el compromiso de cofinanciación, al menos con una cantidad similar, de los proyectos ejecutados en el marco de dichas transferencias.

- El examen de la ejecución del Plan Estatal del Voluntariado ha puesto de manifiesto, junto a los logros anteriormente mencionados, una serie de debilidades o aspectos que, siendo esenciales para la concienciación de la sociedad y la vigorización del voluntariado, apenas han sido desarrollados, y, en consecuencia, no se ha dado un cambio real en esos sectores. Entre éstos cabe destacar los siguientes:

- Redacción excesivamente genérica del Plan, sin mecanismos, sugerencias o instrumentos que permitan a las instancias implicadas llevar a cabo sus objetivos, y sin medios concretos de seguimiento y evaluación de su ejecución y resultados.

- En materia de sensibilización, el Balance recoge los puntos débiles que a continuación se mencionan:

- Escasa visibilidad y difusión de la filosofía, contenidos y proyectos del Plan.

- Acceso muy reducido de las ONG y de las otras instancias implicadas en el fomento del voluntariado, a los medios de comunicación social de carácter general.

- Falta de estudios e investigaciones sobre aspectos cualitativos o cuantitativos del voluntariado y el Tercer Sector

- Escasa incidencia en la concepción del voluntariado como movimiento reivindicativo y transformador de la sociedad.

- El desarrollo del área de promoción, que pretendía crear una cultura de la solidaridad, también adolece de ciertas deficiencias:

- Falta de información sobre la implicación de distintas instancias en la promoción del voluntariado y en el apoyo de las ONG, en particular, de las Corporaciones Locales, a pesar de que éste es el ámbito natural de colaboración, dada la cercanía de los problemas y la orden constitucional a todas las Administraciones de promover la participación de los ciudadanos en el ámbito social, económico, político, etc.

- Escasa implicación de las estructuras y unidades públicas responsables del fomento del voluntariado en materias distintas a la acción social, y, en particular, en la educativa.

- Escasa implicación social de las empresas, dado que no existe aún una cultura de la colaboración no sólo entre el empresariado español, sino también frecuentemente entre las propias ONG.

- Mayor valoración y, en consecuencia, preferencia por los voluntarios, jóvenes en su mayoría, de las ONG de desarrollo y cooperación.

- En relación con el apoyo a las ONG son significativas las siguientes debilidades:

- Falta de adecuación entre las actividades formativas realizadas y las necesidades y demandas de las entidades no lucrativas.

- Pobreza en la oferta formativa para gestores o coordinadores de voluntariado, y para directivos y profesionales de las ONG. 
- Insuficiencia en la financiación pública, que está, además, apenas diversificada por sectores (sólo se conceden para algunas áreas como acción social o cultura) y, en particular, insuficiencia de información sobre las vías y las convocatorias, y sobre los procesos de concesión de numerosas ayudas.

- Escaso desarrollo de las diferentes medidas referidas al apoyo material o a los espacios físicos que las Administraciones Públicas se habían comprometido a ceder a las ONG.

- Debilidad de las estructuras públicas competentes en materia de voluntariado.

- Finalmente en el área de coordinación, que, como queda dicho, es la que se ha desarrollado en menor medida, el Balance apunta los siguientes aspectos:

- Apenas se han formado redes de trabajo que permitan el intercambio de información, la adecuación de las acciones a las verdaderas necesidades de las organizaciones, así como la maximización de los recursos disponibles y de los resultados obtenidos.

- Falta de contacto entre las organizaciones de menor tamaño y las Administraciones, en particular, la Administración General del Estado.

- Escasa coordinación entre ONG y Administraciones Autonómicas, y, cn ocasiones, entre éstas y Corporaciones Locales (Diputaciones, Cabildos, Ayuntamientos, Entidades Locales).

- No ha habido suficiente información, ni comunicación por ambas partes (Administración y ONG) sobre la ejecución de proyectos en desarrollo del Plan.

-- Junto a las consideraciones y datos derivados del Balance del Plan 1997/2000, sc ha de considerar, en segundo lugar, las ideas aportadas durante el proceso de elaboración del nuevo Plan.

- A finales del año 2000, y en paralelo al proceso de redacción del Balance, se comenzó a establecer diversos contactos y reuniones con entidades significativas (ONG de acción social que estaban participando en la valoración del Plan 1997 / 2000, Departamentos Ministeriales, Comunidades Autónomas, Universidades, y la Federación Española de Municipios y Provincias), y les fue enviado un pequeño cucstionario sobre las principales líneas estratégicas que habían de guiar la redacción del Plan 2001/2004, y en que se les solicitaban sugerencias y aportaciones para la elaboración de las nuevas actuaciones y áreas. Además, se recurrió al asesoramiento de algunas figuras expertas en cuestiones sociológicas con el fin de que pudieran dar ideas y propuestas novedosas que enriquecieran y dieran actualidad al texto del Plan.

- Con todo cl material rcunido, la Dirección General de Acción Social, del Menor y de la Familia, del Ministerio de Trabajo y Asuntos Sociales, que ha actuado como coordinadora en la redacción, ejecución y evaluación del Plan Estatal, comenzó a trabajar en el nuevo Plan a principios del año 2001, cuyo borrador fue presentado a lo largo de mes de mayo a las ONG de acción social convocadas inicialmente para su valoración, a la Comisión Técnica Interministerial (compuesta por los departamentos que, en virtud de sus competencias, se encuentran implicados en la materia, como son el Ministerio de Interior, el Ministerio de Justicia, el de Asuntos Exteriores, el Ministerio de Sanidad y Consumo, el Ministerio de Educación, Cultura y Deporte, el de Medio Ambiente y el propio Ministerio de Trabajo y Asuntos Sociales), la Federación Española de Municipios y Provincias, y la Comisión de Seguimiento del Plan (compuesta por los responsables técnicos del voluntariado en las Comunidades Autónomas y las ciudades de Ceuta y Melilla). 
- Posteriormente, en el mes de junio, fue presentado el Plan Estatal del Voluntariado $2001 / 2004$ a la Conferencia Sectorial de Asuntos Sociales y finalmente, se aprobó, como en el caso del anterior Plan, por Acuerdo de Consejo de Ministros el 3 de julio del año 2001.

\section{TÍTULO IV: ÁREAS}

- El Plan Estatal del Voluntariado 2001/2004 pretende dar continuidad a los objetivos y las acciones acometidas en el marco del Plan 1997/2000, pero no como un plan diferente o como un segundo plan, sino como un mismo documento aunque adaptado, por una parte, a una nueva etapa en que nuestras sociedades se harán, paulatinamente, más globalizadas, interdependientes y complejas, y, por otra, a la necesidad de reforzar los aspectos menos desarrollados por el anterior Plan y profundizar en sus logros. De este modo, se posibilita la consecución de objetivos fijados a largo plazo.

- Este carácter continuista ha determinado que se mantuviera, en términos generales, la estructura de áreas, medidas y actuaciones en que se organizaba cl Plan 1997/ 2000 , aunque con algunos cambios: en primer lugar, se sustituye el área de promoción, dado que sus contenidos no quedaban delimitados claramente, dando lugar a ciertas confusiones; en segundo lugar, se introduce el concepto de línea estratégica en sustitución de la denominación de medida, ya que la imposibilidad de actuar en todo lo relativo al movimiento voluntario aconseja dar al Plan un enfoque sectorial que priorice una serie de ámbitos y de acciones orientadas hacia objetivos concretos y a corto plazo; por último, se reduce el número total de actuaciones y se señala, en el caso de que ello sea posible, a qué instancia corresponde su rcalización, con el fin de hacer el Plan más operativo.

- Con estos cambios, se busca configurar un documento más idóneo para hacer frente a las debilidades del anterior Plan y a los desafíos, y para profundizar en los aspectos positivos de lo realizado y en las oportunidades del entorno, con el objetivo de desarrollar el voluntariado en nuestro país, haciendo de él una verdadera forma de participación social y un ejercicio esencial de responsabilidad para los ciudadanos.

\section{SENSIBILIZACIÓN}

- Objetivo: Proporcionar información a toda la sociedad acerca del valor, las oportunidades y la necesidad de participación voluntaria, de acuerdo con las características de cada grupo de edad y con los intereses de cada ciudadano.

- La consecución de este objetivo supone el recurso preferente a una scrie de medios y ámbitos esenciales para el fortalecimiento del movimiento voluntario en España.

- De esos medios destaca, en primer lugar, la difusión de informaciones y conocimientos no sólo sobre qué es el voluntariado, sino también sobre cuáles son los derechos y deberes de los voluntarios, en qué campos trabajan las ONG o qué objetivos y materias aborda el Plan Estatal del Voluntariado 2001 / 2004. En csta misma línea, y de acuerdo con lo señalado por numerosas entidades no lucrativas a lo largo del proceso de elaboración de este documento, la difusión no ha de buscar un mero incremento del número de voluntarios, sino principalmente un voluntariado de calidad, responsable, y plenamente comprometido con la sociedad y con los 
beneficiarios de su trabajo, lo que se puede lograr, entre otros medios, por el reconocimiento de la labor voluntaria y de la figura del voluntario en el seno de cada una de las entidades y en el conjunto de la sociedad.

- Asimismo, es muy importante para la sensibilización fomentar la investigación y el debate en esta materia, ya que se trata de un movimiento particularmente cambiante y heterogéneo que es difícil de delimitar y caracterizar, y en el cual ciertos acuerdos conceptuales y prácticos son fundamentales para su propia pervivencia y para $\mathrm{cl}$ desplicgue de todas las posibilidades que encierra.

- La difusión de informaciones a toda la sociedad implica, necesariamente, un completo desarrolio de las posibilidades de comunicación externa del Tereer Sector, concediendo, en el seno de cada una de las entidades no lucrativas, una atención especial a la profesionalización de estos departamentos y a la realización de acciones significativas para su conocimiento e identificación por cl conjunto de la población, ya que, cn la actualidad, esta identificación se limita, casi cxclusivamente, a grandes organizaciones dedicadas a la cooperación.

- Paralelamente, no cabe duda de que la mayor presencia de las ONG en los medios de comunicación social de carácter general, como radio, prensa y televisión, constituye un elemento imprescindible en la configuración de una nueva imagen social de las ONG, pues también ha de contribuir a su conocimiento e identificación, así como al refuerzo de sus notas críticas y reivindicativas, que en la actualidad están particularmente asociadas con las ONG de cooperación al desarrollo y con las organizaciones medioambientales. Esta presencia en los medios de comunicación ha de difundir los valores y las acciones del voluntariado y las ONG, dando ejemplos y testimonios de cóno encauzar y afianzar los compromisos indjviduales con la sociedad.

- No obstante, existen algunos colectivos y algunos grupos de edad cuya scnsibilización reviste una importancia de primer orden como los niños y los jóvenes, ya que únicamente la impartición generalizada de una educación en valores puede garantizar un futuro solidario y más justo. Y en este contexto, la Universidad desempeña un papel destacado, ya que se trata de una de las instituciones pedagógicas por excelencia, no sólo debido al hecho de que contribuye extraordinariamente a la formación integral de la persona, sino también por su aportación fundamental a la creación de una conciencia crítica en las sociedades mediante la investigación y el estudio, la formulación de nuevas concepciones en materia social y el ofrecimicnto de su bagaje intelectual al debate global que temáticas como las del voluntariado y las $O N G$ pueden suscitar.

- Las personas mayores son otro de los grupos de edad que merece una especial mención, pues se trata de un colectivo cuya participación ciudadana, dado su ticmpo libre, sus experiencias personales, su interés y dedicación, y sus conocimientos profesionales, es de inestimable valor. Asimismo, esa colaboración social puede suponer para los mayores una gran satisfacción personal, un profundo sentimiento de autoestima y la posibilidad de establecer nuevas relaciones.

- Pero la implicación social de las personas mayores, de los universitarios, de los jóvenes y los niños, y de todos los ciudadanos en general, no es necesaria en todos los ámbitos por igual, sino que resulta especialmente interesante en cl trabajo con colectivos en los que, hasta ahora, la colaboración voluntaria ha sido menor como el de enfermos de SIDA, pueblo gitano o personas reclusas y ex reclusas. En esta misma línea, cabe citar también al colectivo de las personas inmigrantes ya que su 
problemática específica y la difusión que de ella han hecho los medios de comunicación social, han dado un importancia capital tanto a la lucha contra la xenofobia y el racismo, como a los esfuerzos de numerosas ONG y entidades públicas por su integración social y laboral. Finalmente, no se ha de dejar de citar la existencia de otros ámbitos en que esta forma de participación social reviste una especial significación como la protección civil, la cultura o la sanidad, y, en particular, el voluntariado medioambiental, rural o local.

\section{I'línea estratégica:}

- Fomento y apoyo de todas aquellas iniciativas que supongan la difusión de las actividades voluntarias, el reconocimiento de los voluntarios y cl debate sobre su concepto y papel social.

\section{Actuaciones:}

1.1. Realización de campañas de concienciación e información a la sociedad sobre la labor desarrollada por ONG y voluntarios, y sobre los valores de solidaridad, justicia y participación en que dicha labor se sustenta.

1.2. Difusión de acciones concretas llevadas a cabo por ONG o por voluntarios que ayuden a un mayor conocimiento y comprensión por la ciudadanía del papel social desempeñado por el Tercer Sector.

1.3. Organización de eventos atrayentes para el conjunto de la sociedad, como exposiciones de fotografía o pósters y carteles, conciertos, festivales de cine, etc., en los que el altruismo y la cooperación sean temáticas centrales.

1.4. Promoción del voluntariado en el ámbito de actuación o con el colectivo específico al que la Organización de Naciones Unidas dedica cada año.

1.5. Celebración del Día Internacional del Voluntariado y de otros Días Internacionales o Mundiales con el fin de dar a conocer diferentes oportunidades de participación voluntaria.

1.6. A poyo, por parte de las entidades públicas y de las entidades privadas lucrativas, a la organización por las ONG de jornadas, seminarios y conferencias para la reflexión y el debate de temas relacionados con el voluntariado.

1.7. Celebración anual de un Congreso Estatal concebido como espacio de encuentro para ONG y voluntarios, y como foro de conocimiento de nuevas experiencias e ideas para el voluntariado.

I.8. Difusión, por parte de la Administración General del Estado, de los contenidos y los logros del Plan Estatal del Voluntariado recurriendo para ello a medios como la elaboración de materiales divulgativos de diverso tipo, la difusión de información por Internet o la celebración de reuniones y jornadas informativas.

1.9. Establecimiento, por parte de las $\mathrm{ONG}$ o de entidades empresariales, de formas de reconocimiento individual y social de los voluntarios, como la difusión de testimonios personales o la concesión de premios.

1. 10. Desarrollo de trabajos de investigación generales que profundicen en el conocimiento de los rasgos actuales del Tercer Sector en sus diferentes ámbitos y en sus tendencias de futuro.

1.11. Realización de estudios sociológicos sobre actitudes y opiniones de la sociedad hacia las ONU, y sobre la motivación y el compromiso de los voluntarios.

1.12. Realización de estudios económicos sobre el valor real de la aportación del voluntariado y sobre el coste que su gestión supone a las organizaciones en que 
colabora.

1.13. Realización de un estudio estadístico y psicosocial para conocer el censo real del voluntariado en tareas de Protección Civil, su distribución territorial, incidencia en la población, perfil y filiación social, motivaciones, expectativas y realidad de este ámbito de colaboración.

1.14. Fomento, por parte de las ONG y las entidades públicas, de un debate abierto y plural acerca del concepto y la práctica del voluntariado, que permita deslindar claramente la figura del voluntario de otras figuras próximas, dando cabida a nuevas experiencias de participación ciudadana, y que permita asimismo determinar el papel social que corresponde a cada sector.

1.15. Fomento de la reflexión, en el marco del Tercer Sector, sobre criterios éticos comunes a considerar en actuaciones externas, como las de tipo comunicacional o de captación de fondos.

1.16. Debate, entre ONG y Administraciones Públicas, acerca de la consolidación de un voluntariado de calidad, responsable y plenamente comprometido con la sociedad y con los beneficiarios de su trabajo.

$2^{a}$ línea estratégica:

- Sensibilización y compromiso de los medios de comunicación social hacia las temáticas relativas a las $O N G$ y el voluntariado, y a los valores que representan.

Actuaciones:

2.1. Fortalecimiento, por parte de las ONG, de sus departamentos de comunicación, por la formación y la profesionalización de los responsables.

2.2. Impulso de la comunicación externa de las ONG mediante diversos medios como la edición de publicaciones periódicas, el diseño de una imagen corporativa o el lanzamicnto de campañas de difusión y publicidad.

2.3. Consolidación de medios escritos, audiovisuales y electrónicos especializados en el voluntariado y en el Tercer Sector.

2.4. Apoyo, por parte de las Administraciones Públicas, a la presencia del Tercer Sector en los medios de comunicación social con el fin de difundir una imagen positiva y realista de las $\mathrm{ONG}$, dar a conocer su labor y sus logros, y proporcionar información a la ciudadanía sobre la posible colaboración en ONG de diverso tamaño y ámbito de actuación.

2.5. Colaboración de los profesionales de la comunicación para que se familiaricen con los temas relativos al voluntariado y la solidaridad, y los aborden en su trabajo, mediante $\mathrm{cl}$ acceso de estos profesionales a información especializada, cursos universitarios de formación y debate, o el contacto personal con profesionales de las ONG y las Administraciones Públicas implicadas en esta materia.

2.6. Promoción del tratamiento, por los medios de comunicación social, de aspectos vinculados con las $\mathrm{ONG}$ en el marco de los debates y programas en los medios audiovisuales, o artículos y reportajes en prensa e Internet.

2.7. Difusión permanente de informaciones referidas al Tercer Sector por las diversas cadenas de televisión existentes, consolidando canales espccializados, creando cspacios televisivos permanentes o por la cesión de espacios para ONG en las cadenas públicas. 


\section{$3^{a}$ línea estratégica:}

- Consolidación de la acción voluntaria en el ámbito educativo.

\section{Actuaciones:}

3.1. Desarrollo, en centros de enseñanza obligatoria, especialmente en los de secundaria y bachillerato, de campañas de sensibilización e implicación de profesores y de otros sectores de la comunidad educativa en acciones de voluntariado y de otras formas de contribución social, por medio de la organización de actos singulares, el acceso a la información existente en Internet, la elaboración de agendas escolares de voluntariado, etc.

3.2. Impulso a la creación, en el marco del Tercer Sector, de fondos documentales e informáticos en materia de voluntariado y educación.

3.3. Edición de material didáctico sobre educación en valores, libros de texto y materiales curriculares, útiles para la formación del profesorado y la enseñanza de los alumnos.

3.4. Realización de proyectos de formación en centros transferidos a Comunidades Autónomas y en los situados en el territorio de gestión del Ministerio de Educación, Cultura y Deporte.

3.5. Desarrollo de proyectos relacionados con el voluntariado y su acción social en los centros escolares, con el fin de que lleguen a formar parte de los proyectos educativos de dichos centros.

3.6. Impulso de la incorporación de los valores y contenidos relativos a las posibilidades de participación y compromiso como materia transversal en la enseñanza reglada.

3.7. Apoyo, por parte de la Administración General del Estado, a la participación de profesores y alumnos en acciones llevadas a cabo en el marco de los programas promovidos desde la agencias europeas, como los programas Sócrates y Leonardo.

3.8. Apoyo a la formación, desde las entidades del Tercer Sector, de agentes educativos voluntarios, como monitores y directores de tiempo libre.

3.9. Realización en los centros de enseñanza de actividades extraescolares cuyos contenidos preparen a los alumnos para futuros compromisos sociales, preferiblemente mediante la intensificación de las relaciones entre ONG y centros.

3.10. Promoción del diseño y lanzamiento de juegos, principalmente electrónicos, basados en la temática social y el voluntariado.

3.11. Creación, en las Universidades, de programas específicos, oficinas o agencias de promoción del voluntariado dedicadas a la sensibilización de la comunidad universitaria, al apoyo de las ONG que desarrollan su trabajo en los campus, y a la coordinación con aquellas instancias que, por su cercanía geográfica o similitud de intereses, puedan ofrecer recursos, experiencias o modelos enriquecedores para los proyectos de la propia Universidad.

3.12. Desarrollo de campañas de sensibilización de la comunidad universitaria hacia la participación social, la colaboración ciudadana y la construcción, en los alumnos, de un espíritu crítico y solidario que contribuya, en cl futuro, a un ejercicio profesional más responsable y justo.

3.13. Impulso a la creación, en el marco universitario, de fondos documentales, bases de datos, hemerotecas, repertorios de investigaciones, y, en general, de cualquier tipo de documento de carácter nacional e internacional que pueda contener 
información significativa para profundizar en la reflexión y el conocimiento del voluntariado en sus diferentes campos de acción.

3. 14. Apoyo a la realización de investigaciones, estadísticas, tesis doctorales, trabajos tutelados o tesinas en materia de ONG y voluntariado.

3.15. Consolidación de los estudios universitarios sobre aspectos económicos, sociales o jurídicos del Tercer Sector, sean asignaturas y materias comprendidas en las titulaciones relacionadas con la salud, la educación o el trabajo social, sean estudios de postgrado y masters, sean seminarios y cursos de verano.

3.16. Apoyo, desde la Universidades, a la plena incorporación de las ONG al ámbito universitario, mediante la facilitación de recursos económicos, materiales o humanos, el asesoramiento por parte del profesorado a las ONG en cuestiones técnicas relacionadas con su práctica docente, la ejecución de proyectos sociales en el campus universitario o proyectos de voluntariado y cooperación en otros ámbitos, o bien mediante la difusión de las necesidades de voluntarios en las ONG.

3.17. Intensificación de las relaciones entre los diferentes campus universitarios, de la cooperación con Ios Ayuntamientos de las localidades en que se encuentran o de las ONG que trabajan en esos municipios, y la colaboración con Administraciones Públicas y cntidades privadas con el fin de favorecer la realización de proyectos conjuntos y el intercambio de conocimientos y prácticas en materia de voluntariado universitario.

$4^{a}$ línea estratégica:

- Consolidación de la acción voluntaria en diferentes grupos de edad y con diferentes colectivos.

\section{Actuaciones:}

4.1. Difusión, por parte de las $O N G$ y de las Administraciones Públicas, de informaciones adecuadas a cada grupo de edad sobre todos los campos, opciones y oportunidades que existen para la colaboración voluntaria.

4.2. Impulso de la participación como voluntarios de colectivos que, por su disponibilidad de tiempo y por su experiencia, pueden hacer una significativa aportación a la actuación de las ONG, como es el caso de las propias personas beneficiarias en los programas, de personas dedicadas a labores domésticas o personas mayores ya jubiladas.

4.3. Incorporación, por parte de las $\mathrm{ONG}$, de jubilados y prejubilados expertos que puedan asesorar a profesionales y voluntarios en materias referidas al funcionamiento de la entidad o a la planificación, ejecución y evaluación de proyectos y programas.

4.4. Fomento de la participación de voluntarios en programas desarrollados por las ONG en materia de acción social, en particular, con aquellos colectivos en los que esta forma de colaboración ha sido menor, como personas afectadas por el VIH/SIDA, minorías étnicas y pueblo gitano y personas reclusas y exreclusas.

4.5. Promoción del voluntariado con personas inmigrantes o pertenecientes a otras culturas, mediante el lanzamiento de campañas o la edición de materiales en que se difunda una información realista acerca de su perfil humano y problemática, con el fin de crear en la sociedad española una imagen positiva de este colectivo.

4.6. Creación, en el marco del Tercer Sector, de fondos documentales e informativos 
referidos a la enseñanza de la lengua y cultura españolas que puedan ser de utilidad, como material didáctico y de apoyo, a los voluntarios que colaboran en programas de enseñanza e integración social de colectivos inmigrantes.

4.7. Promoción del voluntariado en aquellos campos en que su participación es de especial importancia para la resolución de los problemas clave en cl conjunto nacional o internacional como es el caso de la cooperación al desarrollo, la sanidad, la cultura o el deporte.

4.8. Fomento de la elaboración y aplicación de planes sectoriales de voluntariado en espacios protegidos y ámbitos afines, siguiendo el modelo del Plan de voluntariado en Parques Naturales.

4.9. Impulso, por parte de las Administraciones Públicas, de un afianzamiento de los vínculos entre los Parques Naturales y las comunidades locales en que están situados, con el fin de lograr un desarrollo comarcal duradero.

4.10. Promoción del voluntariado en Protección Civil entre grupos de edad tradicionalmente alejados de este campo, como la población adulta o la jubilada.

4.11. Consolidación de un voluntariado rural por medio de la realización y difusión de estudios, el lanzamiento de campañas, el apoyo a las ONG que trabajan en el mundo rural y a las asociaciones de vecinos, etc.

4.12. Estímulo, por parte de las Administraciones territoriales competentes o por las asociaciones de entidades locales, a la creación de Planes Municipales de voluntariado, con el fin de consolidar en España un movimiento voluntario de fuerte implante local.

4.13. Realización de acciones divulgativas y formativas referidas a las metas y contenidos de los Planes Municipales de voluntariado.

4. 14. Apoyo, desde la Federación Española de Municipios y Provincias, a la elaboración e implementación de políticas locales dirigidas al fomento de la participación ciudadana y el voluntariado.

\section{APOYO}

- Objetivo: Comprometer al sector público, al sector lucrativo y al propio Tercer Sector en la modernización de las ONG y en su dotación, adecuadas al destacado papel social que han de desempeñar.

- Todos los sectores han de asumir, de diferente modo, la responsabilidad que les corresponde en respuesta a los nuevos desafíos y necesidades que los cambios sociales y la globalización traerán, y, en particular, con su apoyo a las organizaciones de voluntariado, aspecto éste en el que cabe señalar algunas propuestas y sugerencias de actuación.

- Por una parte, son especialmente significativas las propuestas relativas a la modernización de las entidades, tanto de su estructura y organización, en virtud de la aplicación de valores democráticos y participativos, como de su funcionamiento y gestión, tomando de la empresa privada aquellos principios, sistemas y prácticas que les puedan resultar más útiles para desarrollar una labor efectiva y de calidad.

- Por otra, el fortalecimiento del movimiento voluntario español exige, más allá de la consolidación del Tercer Sector, su desarrollo pleno, para lo cual le es necesario contar con unos medios humanos, económicos y materiales y unos espacios físicos suficientes y adecuados a sus cometidos, un ordenamiento jurídico adaptado 
a la nueva sociedad del bienestar así como información actualizada y fluida, ya que ésta ha pasado a ser, hoy en día, el instrumento clave para la dirección eficiente de las organizaciones.

- Por último, la creación de una cultura empresarial sensible a los problemas sociales, la inclusión de la participación y la colaboración ciudadana en el marco de la gestión de recursos humanos, y el apoyo económico que las empresas pueden brindar a las ONG, bien optando prioritariamente por sus servicios, bien ofreciendo sus propias prestaciones y productos comerciales en unas condiciones más favorables, constituyen elementos de una relación que puede ser beneficiosa tanto para la empresa como para el Tercer Sector.

- Para las metas del Tercer Sector, dicha colaboración puede suponer la presencia de unos profesionales que les ayuden extraordinariamente a desarrollar su trabajo habitual, puede suponer también la diversificación de sus ingresos, y con ella, lograr independencia, así como la posible realización de actuaciones de mayor envergadura y mayor solidez. Para el sector empresarial la colaboración puede significar la opción por una estrategia de maximización de los márgenes de beneficio que se dan en la actualidad, el aumento de la utilidad de un bien o un servicio y de la satisfacción de los clientes al elegirlo y consumirlo, así como un refuerzo de la motivación del personal y el conocimiento de una forma distinta de trabajar en que Ia flexibilidad, la orientación hacia los objetivos y el trabajo en equipo son prioritarios, afianzando, en definitiva, la configuración de una imagen más humanizada de la cmpresa ante su propio personal y ante la sociedad.

\section{$5^{x}$ línea estratégica:}

- Apoyo a la modernización organizativa y funcional de las entidades del Tercer Sector.

\section{Actuaciones:}

5.1. Apoyo, por parte de las Administraciones Públicas y de las ONG dedicadas al asesoramiento de otras entidades del Tercer Sector, al estudio e implementación de procesos de modernización funcional y organizativa en cada entidad.

5.2. Fortalecimiento, principalmente en las ONG de menor tamaño, de unas estructuras mínimas que les permitan desarrollar su labor con éxito, mediante la consolidación de relaciones con otras entidades de características y ámbito similares.

5.3. Aprovechamiento, por parte de las $\mathrm{ONG}$, de las posibilidades que ofrecen las nuevas tecnologías para el fortalecimiento de sus estructuras y el desarrollo de sus proyectos, como la exploración de nuevas formas de captación de fondos, el voluntariado virtual, la formación a distancia, etc.

5.4. Aprovechamiento, por parte de las $\mathrm{ONG}$, de las posibilidades que ofrecen las nuevas tecnologías para el fortalecimiento de sus estructuras y el desarrollo de sus proyectos, como la exploración de nuevas formas de captación de fondos, el voluntariado virtual, la formación a distancia, etc.

5.5. Facilitación, por parte de las Administraciones Públicas y las entidades privadas, de la presencia activa de las ONG en Internet, mediante el alojamiento de sus propias páginas, la consolidación de portales de voluntariado y colaboración social, o el aprendizaje y la extensión del uso de nuevas tecnologías de la comunicación en el marco de cada entidad. 


\section{6“ínea estratégica:}

- Dotación de recursos humanos, económicos y de otro tipo adecuada a las acciones de cada $O N G$.

\section{Actuaciones:}

6.1. Refuerzo de la capacidad de las ONG para dar cabida en sus estructuras y funcionamiento a los ciudadanos que deseen colaborar en ellas como voluntarios, respetando el perfil singular de cada uno y aprovechando sus potencialidades para cumplir los diferentes cometidos de la entidad.

6.2. Promoción de proyectos para la detección de las verdaderas necesidades y demandas del Tercer Sector en materia de formación, y adaptación a ellas de la oferta de entidades de voluntariado, Administraciones Públicas y otras instancias dedicadas a la capacitación de los trabajadores de las ONG.

6.3. Impartición, por parte de las $\mathrm{ONG}$, de acciones formativas para los voluntarios, tanto con carácter genérico, como relativas al sector concreto de especialización de la entidad, con el fin de lograr una mayor eficacia del voluntariado.

6.4. Realización de acciones formativas específicas en materia de Protección Civil

6.5. Celebración de cursos de voluntariado en materia de Protección Civil para la autoprotección escolar.

6.6. Elaboración, por parte de las ONG, de planes conjuntos de formación de voluntarios para las diferentes áreas de acción en que desarrollan su labor, y creación, en el marco del Tercer Sector, de centros de capacitación para voluntarios y profesionales.

6.7. Profesionalización, por parte de las $O N G$, de la figura del coordinador de voluntariado, responsable de informar a los voluntarios sobre sus propios cometidos y sobre el conjunto de la organización, guiarles en su proceso de incorporación, dirigirles en la ejecución de su trabajo y motivarles de modo que se afiance su compromiso.

6.8. Realización, por parte de las $\mathrm{ONG}$, de acciones formativas adecuadas para los coordinadores o gestores de voluntariado.

6.9. Celebración de cursos de formación de formadores en diversas materias.

6. I0. Promoción, por parte de las $\mathrm{ONG}$, de principios y prácticas democráticas en el seno de sus estructuras que permitan a voluntarios y profesionales participar en el diseño de los programas y planes de la entidad.

6.11. Formación de los directivos, profesionales y gestores de ONG en sus respectivas tareas, poniendo un énfasis especial en la formación relativa a nuevas tecnologías, cuestiones tributarias e impositivas, aspectos jurídicos, contables y aspectos relacionados con la captación de fondos.

6.12. Apoyo, por parte de las Administraciones Públicas y las entidades empresariales, para que las ONG cuenten con una dotación adecuada de medios técnicos y materiales, y muy especialmente de equipamientos informáticos y electrónicos actualizados.

6.13. Profundización en los aspectos legales relacionados con la facilitación de la labor desarrollada por ONG y voluntarios, y reforma, en lo que sea necesario, del marco jurídico del Tercer Sector.

6.14. Diversificación de las fuentes de financiación de asociaciones y fundaciones, vigorizando aquéllas que permitan la realización de proyectos a largo plazo, la independencia de las ONG respecto a otras instancias, y que conlleven una 
mayor colaboración ciudadana.

6. I5. Apoyo cconómico, desde las Administraciones Públicas, para la sensibilización y promoción del voluntariado y las $\mathrm{ONG}$, por sectores y ámbitos territoriales.

6.16. Difusión general de informaciones sobre las convocatorias y la concesión de subvenciones nacionales e internacionales a este sector.

6.17. Consolidación de instrumentos de información útiles para la dirección y gestión de las entidades del Tercer Sector, como bases de datos informatizadas, guías de bucnas prácticas, fondos documentales, páginas web en Internet dedicadas a las ONG, guías territoriales y sectoriales de voluntariado, centros de información general, etc.

6.18. Apoyo, por parte de las Administraciones Públicas, al refuerzo de las relaciones entre entidades mediante la cesión puntual de espacios físicos para las reuniones $o$ actos de varias $\mathrm{ONG}$.

\section{$7^{a}$ línea estratégica:}

- Consecución de la implicación social de la empresa.

Actuaciones:

7.1. Apoyo, por parte de las Administraciones Públicas, a la consolidación de nuevas concepciones como las de empresa ética y voluntariado corporativo, mediante la orientación de las culturas empresarial y del Tercer Sector hacia valores y prácticas diferentes, basados en la corresponsabilidad y la colaboración intersectorial.

7.2. Incorporación de la acción social externa a la negociación colectiva, de modo que sea considerada en diversos aspectos de la gestión de personal, como la selección, la formación o la preparación para la jubilación.

7.3. Valoración, por parte de los responsables de la gestión de recursos humanos, de la experiencia adquirida por los trabajadores de las ONG, a efectos de su incorporación en la empresa.

7.4. Promoción, a través de las organizaciones empresariales y sindicales, de programas de información y capacitación del personal basados en la cooperación con ONG, por medio de la asignación de un grupo de empleados a una ONG para completar su formación, o por la cesión por la empresa, durante un plazo determinado o por unas horas, de alguno de sus trabajadores cualificados para que se implique en proyectos concretos de una $\mathrm{ONG}$

7.5. Apoyo, por las empresas, a las iniciativas sociales promovidas por los propios empleados, como la donación de parte del salario mensual, la creación de fórmulas de reconocimiento entre ONG con las que colaboren los empleados, la constitución por los empleados de una entidad de acción social propia, ctc.

7.6. Fomento de la inclusión, en los programas empresariales de preparación para la jubilación, de informaciones relativas a las diversas posibilidades de colaboración ciudadana y voluntariado, considerando la experiencia y el tiempo libre de los trabajadores jubilados y el enriquecimiento personal que la participación social les puede suponer.

7.7. Promoción del patrocinio y el mecenazgo de acciones de contenido social por parte de las entidades empresariales, financiando proyectos concretos o la actividad global de una ONG mediante compromisos de colaboración permanente. 
7.8. Difusión de las diversas posibilidades de colaboración relacionadas con el marketing con causa, el marketing corporativo y otras formas de comunicación externa que, asimismo, establezcan relaciones de productos y servicios con causas de interés social.

7.9. Cesión, por parte de las entidades empresariales, de servicios de apoyo técnico así como de equipamientos, infraestructuras y otros bienes materiales a entidades del Tercer Sector con las que colaboren.

7.10. Sensibilización de los responsables de empresas para que contraten la fabricación de productos o la prestación de servicios externos preferentemente con entidades no lucrativas y con empresas de iniciativa social.

7.11. Difusión de informaciones, entre las entidades empresariales, acerca de las peculiaridades de gestión y actuación de las organizaciones no lucrativas, con el fin de que oferten a las ONG sus servicios o productos a un menor precio o con unas condiciones más favorables, o bien que diseñen productos y prestaciones adaptadas a esas peculiaridades.

\section{COORDINACIÓN}

- Objetivo: Fortalecer la colaboración entre Administraciones Públicas y ONG, prestando una especial atención a su implicación en las estructuras y redes nacionales e internacionales de cooperación.

- La consecución de este objetivo supone el recurso preferente a una serie de medios, sugerencias y propuestas de acción esenciales para el fomento de la coordinación de todas las instancias, públicas y privadas, lucrativas o no, implicadas en la promoción del voluntariado y del Tercer Sector.

- El primer ámbito en que se ha de fortalecer la colaboración es el ámbito lucrativo, ya que la intensificación de las relaciones entre ONG ha de suponer un importante intercambio de experiencias y conocimientos sobre necesidades y problemas comunes, demandas, y vías de solución, así como un punto de partida fundamental desde cl que alcanzar compromisos y acuerdos sobre aspectos conceptuales y prácticos claves para el desarrollo pleno del Sector. En la misma línea destaca asimismo el apoyo, principalmente por parte de las Administraciones Públicas a las federaciones de $\mathrm{ONG}$, confederaciones y estructuras de diverso tipo que, precisamente, cncarnan esta coordinación, así como el apoyo de aquellas entidades especialmente dedicadas a la concienciación de la sociedad y la promoción del voluntariado, debido, por una parte, al significado de su labor y, por otra, a que cn muchas de ellas el trabajo voluntario mismo no tiene una especial significación.

- Paralelamente, también se considera esencial el refuerzo de las unidades administrativas que tienen asignada la función específica de fomento de las ONG y de la participación ciudadana, con el fín de constituirlas en referente público en sus diferentes ámbitos territoriales y comunidades, bien mediante la dotación de recursos humanos, materiales y económicos adecuados a las funciones encomendadas, bien mediante la difusión en el Tercer Sector de una completa información acerca de los servicios prestados por las unidades y de los proyectos realizados en pro de las $\mathrm{ONG}$.

- A su vez, se ha de intensificar la colaboración entre unidades y responsables públicos dedicados al voluntariado en el seno de cada Administración y en el de posibles estructuras o encuentros inter-administrativos, de modo que la promo- 
ción del voluntariado, concebido como vía preferente de participación en la construcción de la Sociedad del Bienestar, sea un proyecto común, aunque a distinto nivel y con diferentes manifestaciones (sanitaria, educativa, medioambiental, social, penitenciaria, etc).

- No obstante, la cobertura de todas las necesidades de los colectivos y de los campos de actuación voluntaria exige una coordinación más amplia que alcance a ONG, Administraciones Públicas, Universidades, entidades empresariales y, en general, cuantos individuos $\mathrm{e}$ instancias se hallen implicadas en la acción y la ayuda desinteresadas, aunque priorizando, como no podía ser menos, aquéllas que por su ámbito territorial o por su campo de actuación se puedan considerar como más próximas a cada entidad, ya que es ésta la vía clave para dar una atención integral a cada grupo y a cada problema.

- La implicación de las distintas instancias españolas en redes europeas y, en general, en redes internacionales constituye una respuesta inaplazable al desafío de la globalización, de la acción en un entorno que se hace cada vez más grande, con mercados que abarcan casi todo el conjunto mundial, necesidades que afectan a la mayor parte de la humanidad, organizaciones y estrategias globales. Y ello da lugar a que el núcleo último de dicho desafío sea la cooperación al desarrollo del Tercer y Cuarto Mundo, al crecimiento sostenible de los países y de los sectores más marginados de las sociedades contemporáneas, incrementando la riqueza y su distribución entre la población, y respetando el medio ambiente, los derechos humanos y las singularidades socioculturales de cada grupo y nación.

\section{8alínea estratégica:}

- Fortalecimiento de las relaciones de coordinación entre ONG.

\section{Actuaciones:}

8.1. Impulso del trabajo en red entre ONG, en particular entre las de menor tamaño, para el desarrollo conjunto y complementario de actuaciones por sectores o colectivos similares, con el fin de dar una cobertura completa a los problemas de cada ámbito territorial, cada campo y cada beneficiario, y de fortalecer el Tercer Sector.

8.2. Edición y dilusión, por parte de las $\mathrm{ONG}$, de documentos comunes que puedan facilitar la labor de todas aquellas entidades que trabajan en un mismo sector.

8.3. Intercambio de experiencias y conocimientos entre $O N G$ por medio de reuniones, estructuras de coordinación, intercambio de voluntarios, intensificación de las relaciones entre los responsables o coordinadores de voluntariado, creación de plataformas de comunicación, etc.

8.4. Apoyo, por parte de las Administraciones públicas, a la consolidación y funcionamiento de las estructuras de coordinación (federaciones y confederaciones) y plataformas del Tercer Sector, a nivel local, autonómico y estatal.

8.5. Apoyo, por parte de las Administraciones Públicas, al nacimiento y consolidación de organizaciones dedicadas específicamente a la promoción del voluntariado en sus distintos ámbitos de acción.

\section{$9^{a}$ línea estratégica:}

- Vigorización de la coordinación en el ámbito administrativo y de la participación de las ONG en la prestación de scrvicios públicos. 


\section{Actuaciones:}

9.1. Fortalecimiento de las unidades administrativas dedicadas a la promoción del voluntariado y al apoyo de las ONG.

9.2. Difusión de informaciones sobre los recursos y las funciones encomendadas a las unidades administrativas para el voluntariado, con el fin de darles una mayor visibilidad ante el sector asociativo y el conjunto del sector público.

9.3. Apoyo, en el marco de cada Administración Pública, a la interrelación de las unidades administrativas con otras unidades pertenecientes a Departamentos o Conserjerías diferentes.

9.4. Fomento de la cooperación entre entidades locales, y de éstas con sus respectivas Comunidades Autónomas, por medio del intercambio de experiencias y conocimientos en materia de voluntariado, en el marco de reuniones periódicas, órganos o redes de colaboración, y por medio de la ejecución de proyectos conjuntos.

9.5. Potenciación del trabajo coordinado entre las Comunidades Autónomas y los Ayuntamientos en materia de servicios sociales.

9.6. Fortalecimiento de la coordinación entre Comunidades Autónomas para el desarrollo de acciones comunes y la difusión de informaciones sobre actuaciones y estructuras dedicadas al voluntariado con el fin de mejorar la organización y funcionamiento de cada Comunidad.

9.7. Promoción de estructuras e instrumentos administrativos para la cooperación entre las Comunidades Autónomas y la Administración General del Estado, y mejora y agilización del funcionamiento de los ya existentes.

9.8. Potenciación a la creación y mantenimiento de órganos de colaboración entre Administraciones Públicas y ONG en los diferentes ámbitos territoriales, para la participación del sector asociativo en el desarrollo de las políticas públicas referidas a los distintos campos de actuación.

9.9. Apoyo a la participación de las ONG en la prestación de servicios públicos mediante la ejecución de proyectos conjuntos, el acceso de las asociaciones y fundaciones a centros públicos, o la opción preferente, por parte de los responsables administrativos, hacia la contratación de organizaciones de voluntariado en lugar de entidades empresariales.

\section{$10^{u}$ línea estratégica:}

- Incorporación de las ONG y de las unidades administrativas al entorno internacional.

Actuaciones:

10.1. Fomento de las relaciones de las Administraciones Públicas españolas con las de otros países y organismos internacionales, principalmente comunitarios, con el fin de poder ofrecer al Tercer Sector una completa visión sobre la situación global del voluntario, problemas concretos y vías de solución.

10.2. Difusión de informaciones de carácter intemacional que permita a las ONG tener un conocimiento actualizado de las principales entidades internacionales existentes en cada sector y colectivo, de las redes de mayor consolidación y los cventos internacionales de más importancia.

10.3. Implicación y participación de las $\mathrm{ONG}$ en redes internacionales, de acuerdo con su propia fïlosofía y ámbito de actuación, en particular, en aquellas áreas 
que son más cercanas geográficamente a España como el continente europeo, Ibcroamérica y el Mediterráneo.

10.4. Apoyo, por parte de las Administraciones Públicas, a las campañas de sensibilización desarrolladas por ONG en pro de una mayor concienciación y solidaridad de la sociedad española hacia los problemas del Tercer y Cuarto Mundo.

10.5. Mantenimiento de la colaboración española al Programa de Voluntariado de Naciones Unidas.

10.6. Impulso de los programas de partenariado en el ámbito europeo para el desarrollo de acciones formativas y de investigación en materia de voluntariado.

\section{TÍTULO V: SEGUIMIENTO Y EVALUACIÓN DEL PLAN ESTATAL DEL VOLUNTARIADO}

- Una de las debilidades del Plan 1997/2000 que ha sido señalada en el Balance se refiere a su redacción genérica, dado que en el momento de su elaboración se consideró que lo más interesante sería recoger una enumeración de objetivos, deseos y principios, y no la incorporación de instrumentos concretos de seguimiento. y evaluación que permitieran conocer quizá no tanto su grado d ejecución, ya que esto es sencillo por medio de los informes que se han venido realizando durante los años anteriores, sino principalmente sus resultados periódicos, sus logros y su impacto global en la sociedad y en el movimiento voluntario.

- Por ello, se ha determinado la inclusión en el presente Plan de un apartado específico dedicado a la cvaluación, en que se da a conocer la periodicidad de las evaluaciones, las posibilidades de difusión de sus conclusiones, la metodología y fuentes de información, así como los indicadores que permitan medir y, en cierto modo, facilitar datos complementarios para conocer la evolución del Tercer Sector en nuestro país.

- En cuanto a la periodicidad de las valoraciones, como se hizo, aunque informalmentc, con cl anterior Plan Estatal, también se prevé llevar a cabo un seguimiento anual de lo realizado en virtud del Plan, que se cierre al finalizar cada uno de los años naturales que abarca el nuevo documento, y dar a conocer sus conclusiones bien en un acto específico; bien durante la celebración de algún evento suficientemente significativo como para que estos resultados tengan el adecuado eco, como son, entre otros, el Día Internacional del Voluntariado o la organización del Congreso Estatal que se viene celebrando desde 1997.

- Para la realización de este seguimiento, así como para llevar a cabo la evaluación final a la conclusión de los cuatro años, se invitar a todas las instancias interesadas, sean las que hasta ahora se han incluido en las valoraciones, sean otras instancias nuevas, pero cuya acción puede ser de especial relevancia (como medios de comunicación social, entidades empresariales o instituciones educativas) para que den a conocer los proyectos y actuaciones realizadas que mantengan alguna relación con los contenidos del Plan Estatal, con el fin de poder analizar su información y elaborar un documento único que dé una visión de conjunto sobre la promoción del voluntariado en España. 


\section{INDICADORES DE SENSIBILIZACIÓN}

- El objetivo propuesto para el área de sensibilización es el siguiente: Proporcionar información a toda la sociedad acerca del valor, las oportunidades y la necesidad de participación voluntaria, de acuerdo con las características de cada grupo de edad y con los intereses de cada individuo.

- Este objetivo se prevé valorar por medio de los siguientes indicadores:

\section{Indicadores Finalistas}

$\mathrm{N}^{\circ}$ de personas que colaboran como voluntarias en ONG y AAPP.

$\mathrm{N}^{\circ}$ medio de horas que colaboran.

$\mathrm{N}^{\circ}$ medio de años que colaboran.

Porcentaje de población global que conoce qué es el voluntariado.

1. Fomento y apoyo de todas aquellas iniciativas que supongan la difusión de las actividades voluntarias, el reconocimiento de los voluntarios y el debate sobre su concepto y papel social:

\begin{tabular}{|l|} 
Indicadores de Proceso \\
$N^{\circ}$ de campañas de sensibilización realizadas en materia de voluntariado. \\
$N^{\circ}$ de actos realizados en celebración de hechos o días conmemorativos. \\
$N^{o}$ de actos o materiales promovidos para dar a conocer el Plan Estatal. \\
$N^{\circ}$ de nuevas iniciativas para reconocer la labor de ONG o voluntarios. \\
$N^{o}$ de estudios realizados sobre aspectos generales o en algún campo específico. \\
$N^{o}$ de documentos o actos conjuntos que aborden temas novedosos o polćmicos.
\end{tabular}

2. Sensibilización y compromiso de los medios de comunicación social hacia las temáticas relativas a las ONG y el voluntariado, y los valores que representan:

Indicadores de Proceso
$N^{\circ}$ de nuevos medios especializados en prensa, radio, televisión e Internet.
$\mathrm{N}^{\circ}$ de programas, artículos o reportajes emitidos en medios sociales generales.
$\mathrm{N}^{\circ}$ de proyectos realizados para la sensibilización de medios y profesionales.

3. Consolidación de la acción voluntaria en el ámbito educativo:

Indicadores de Proceso
$\mathrm{N}^{\circ}$ de campañas de sensibilización realizadas en el marco educativo.
$\mathrm{N}^{\circ}$ de programas formativos de profesores/alumnos vinculados con esta temática.
$\mathrm{N}^{\circ}$ de ONG con acuerdos con centros educativos.
$\mathrm{N}^{\circ}$ de nuevas agencias o programas universitarios de voluntariado.
$\mathrm{N}^{\circ}$ de nuevas asignaturas universitarias, estudios y cursos sobre ONG.
$\mathrm{N}^{\circ}$ de ONG con acuerdos con centros universitarios.

4. Consolidación de la acción voluntaria en diferentes grupos de edad y con diferentes colectivos:

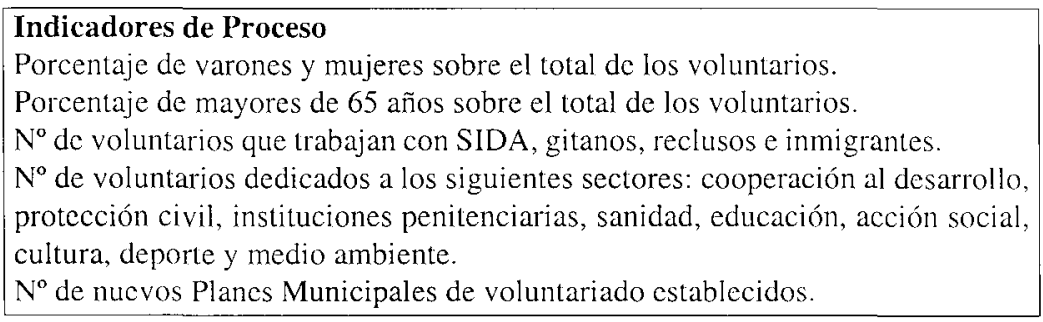




\section{INDICADORES DE APOYO}

- El área de apoyo persigue la siguiente meta: Comprometer al sector público, al privado lucrativo y al propio Tercer Sector en la modernización de las ONG y en su dotación, adecuadas al destacado papel social que han de desempeñar.

- El seguimiento y evaluación de esta meta se prevé conocer mediante diversos indicadores:

\begin{tabular}{l} 
Indicadores Finalistas \\
$\mathrm{N}^{\circ}$ de nuevas ONG inscritas en el Registro de Asociaciones. \\
Porcentaje del PIB correspondiente a las ONG / Tercer Sector. \\
$\mathrm{N}^{\circ}$ medio de voluntarios por entidad. \\
$\mathrm{N}^{\circ}$ medio de socios por entidad. \\
\hline
\end{tabular}

5. Apoyo a la modernización organizativa y funcional de las entidades del Tercer Sector:

Indicadores de Proceso
$\mathrm{N}^{\circ}$ de proyectos orientados hacia la modernación de las ONG.
$\mathrm{N}^{\circ}$ de acciones orientadas hacia la presencia de las ONG en Internet.

6. Dotación de recursos humanos, económicos y de otro tipo adecuada a las acciones de cada ONG:

\begin{tabular}{|l|}
\hline Indicadores de Proceso \\
$\mathrm{N}^{\circ}$ de acciones formativas de voluntarios. \\
$\mathrm{N}^{\circ}$ de acciones formativas de voluntarios en las siguientes materias: cooperación al \\
desarrollo, protección civil, instituciones penitenciarias, sanidad, educación, ac- \\
ción social, cultura, deporte y medio ambiente. \\
$\mathrm{N}^{\circ}$ de ONG que han creado la figura del coordinador o gestor de voluntariado. \\
$\mathrm{N}^{\circ}$ de acciones formativas para profesionales, directivos y gestores de ONG. \\
$\mathrm{N}^{\circ}$ de voluntarios que ocupan puestos directivos. \\
Gasto medio dedicado por las ONG a programas de voluntariado. \\
$\mathrm{N}^{\circ}$ de instrumentos de información creados o editados. \\
\hline
\end{tabular}

7. Consecución de la implicación social de la empresa:

\begin{tabular}{|l|} 
Indicadores de Proceso \\
$\mathrm{N}^{\circ}$ de proyectos realizados para la sensibilización del empresariado. \\
$\mathrm{N}^{\circ}$ de acuerdos de colaboración puntual o permanente entre empresas y ONG. \\
$\mathrm{N}^{0}$ de proyectos orientados al patrocinio empresarial de acciones solidarias. \\
$\mathrm{N}^{\circ}$ de productos o servicios prestados por empresas a ONG. \\
\hline
\end{tabular}

\section{INDICADORES DE COORDINACIÓN}

- El objetivo propuesto para el área de coordinación es el siguiente: Fortalecer la colaboración entre Administraciones Públicas y ONG, prestando una especial atención a su implicación en las estructuras y redes nacionales e internacionales de cooperación.

- Estc objetivo se prevć valorar por medio de los siguientes indicadores:

Indicadores Finalistas
Porcentaje de integración de ONG en coordinadoras, federaciones, confederacio-
nes, plataformas, ...
$\mathrm{N}^{\circ}$ de consejos territoriales creados.
Porcentaje de ONG y AAPP integradas en redes internacionales.


8. Fortalecimiento de las relaciones de coordinación entre ONG:

\section{Indicadores de Proceso}

$\mathrm{N}^{\circ}$ de proyectos ejecutados conjuntamente, al menos, por dos cntidades.

$N^{0}$ de nuevas plataformas o estructuras de coordinación creadas.

9. Vigorización de la coordinación en el ámbito administrativo y de la participación de las ONG en la prestación de servicios públicos:

\section{Indicadores de Proceso}

$N^{\circ}$ medio de empleados en unidades administrativas (generales y autonómicas).

$\mathrm{N}^{\circ}$ de proyectos realizados conjuntamente por distintas Administraciones.

$N^{\circ}$ de proyectos de coordinación entre ONG y Administraciones Públicas.

10. Incorporación de las ONG y de las unidades administrativas al entorno internacional:

\section{Indicadores de Proceso}

$\mathrm{N}^{\circ}$ de ONG españolas integradas en redes internacionales.

$N^{o}$ de Administraciones españolas integradas en redes internacionalcs. 